\title{
The interplay between miR-1245a and BRCA2 in colorectal cancer
}

\author{
Andreas Scorilas, Konstantina Panoutsopoulou, Panagiotis Tsiakanikas \\ Department of Biochemistry and Molecular Biology, Faculty of Biology, National and Kapodistrian University of Athens, Athens, Greece \\ Correspondence to: Prof. Andreas Scorilas. Department of Biochemistry and Molecular Biology, Faculty of Biology, National and Kapodistrian \\ University of Athens, Panepistimiopolis, 15701 Athens, Greece. Email: ascorilas@biol.uoa.gr. \\ Provenance and Peer Review: This article was commissioned by the editorial office, Annals of Translational Medicine. The article did not undergo \\ external peer review. \\ Comment on: Pan Z, Gan W, Liang C, et al. miR-1245a promotes the proliferation and invasion of colon adenocarcinoma by targeting BRCA2. Ann \\ Transl Med 2019;7:777.
}

Submitted May 07, 2020. Accepted for publication May 25, 2020.

doi: $10.21037 /$ atm-2020-113

View this article at: http://dx.doi.org/10.21037/atm-2020-113

Epidemiological evidence, presented by Global Cancer Observatory (GCO), rank colorectal adenocarcinoma (CRC) in the $3^{\text {rd }}$ place of the most diagnosed malignant neoplasms and the $2^{\text {nd }}$ cause of cancer-related deaths worldwide, in both sexes, while World Health Organization (WHO) projections illustrate an international $40 \%$ increase of CRC incidences and deaths until 2030. CRC is characterized by great intra- and inter-patient heterogeneity as a result of its complex genetic landscape. The majority of the incidences $(\sim 70 \%)$ are sporadic cases, whereas the rest $30 \%$ refer to an underlying familial predisposition or an established genetic syndrome (1).

Sporadic CRC is guided by the stepwise progression of benign neoplasms to malignant tumors using two distinct pathways, (I) the typical adenoma-carcinoma pathway and (II) the serrated pathway, which includes a variety of serrated polyps/adenomas as precursor lesions of CRC. In the conventional adenoma-carcinoma model, the accumulation of genetic alterations is mediated by the molecular mechanisms of chromosomal instability (CIN) and/or microsatellite instability (MSI). In this regard, early mutations of oncogenes and tumor-suppressor genes, such as $A P C$ and $K R A S$, respectively, are key initiators during the development of CIN, whereas MSI is guided by mutations in genes involved in DNA mismatch repair (MMR). On the contrary, serrated lesions advance to CRC through the molecular pathway of MSI and/or acquisition of the CpG island methylator phenotype (CIMP). In the latter, which is characteristic of serrated lesions, the genomic instability of malignant and premalignant lesions is driven by aberrant
DNA methylation, specifically in promoter regions of regulatory genes, resulting in their epigenetic silencing. Another common characteristic of some serrated lesions, is the activation of the mitogen activated protein kinase (MAPK) pathway in the initial phase of their development, which is mediated by activating mutations of $B R A F$ and KRAS proto oncogenes respectively $(2,3)$.

The release of the ENCODE project in 2010 unveiled that more than $75 \%$ of the genome is transcribed into noncoding RNAs (ncRNAs) that have gained the ever-growing attention of the researchers due to their active involvement in the majority of cellular processes and tumorigenesis as well as their potential clinical role as attractive candidate biomarkers for diagnosis, prognosis and treatment response of human malignancies (4-6). MicroRNAs (miRNAs), which represent a well-studied category of ncRNAs, are $\sim 22 \mathrm{nt}$ long highly conserved ncRNAs that have arisen as the most potent regulators of gene expression at the posttranscriptional level. Transcribed by RNA polymerase II as mono- or poly-cistronic hairpin-containing primary precursors, they are further processed in the nucleus by the RNase III-type endonuclease Drosha and the dsRNAbinding protein, DiGeorge syndrome Critical Region Gene 8 (DGCR8) to release the stem loop precursor miRNA (pre-miRNA). Following nuclear export via Exportin 5 (XPO5), the pre-miRNA is cleaved by the RNase IIItype endonuclease Dicer and forms a duplex, comprising of the mature miRNA sequence and its passenger strand. Thereafter, the mature miRNA, interacting with Argonaute (AGO) proteins, directs the miRNA-induced silencing 
complex (miRISC) to target mRNAs with complementary regions in the 3' untranslated region (3'-UTR) inducing gene silencing via translational repression as well as deadenylation and decay of the target mRNAs $(7,8)$. Apart from the canonical pathway, miRNA biogenesis pathways independent of Drosha and/or Dicer have been, also, reported (9). Strikingly, miRNAs are involved in almost all aspects of cellular processes and mounting evidence implicates them in cancer initiation and progression, through their ability to act as oncogenes or tumorsuppressors $(10,11)$. Ultimately, miRNAs have emerged as promising biomarkers for diagnosis, prognosis and disease course monitoring (12-14).

miR-1245a is encoded by MIR $1245 \mathrm{~A}$ gene in the $2 \mathrm{q} 32.2$ locus. Recently, it has been revealed that miR1245 a directly targets $B R C A 2$ 3'-UTR, and that MYCmediated upregulation of miR-1245a leads to BRCA2 suppression, and thus to DNA damage induction and genomic instability in primary breast tumors (15). Likewise, miR-1245a-mediated downregulation of BRCA2 promotes lung cancer progression in vitro, whilst increased BRCA2 expression, through hsa-circ-0046264-mediated downregulation of miR-1245a enhances apoptosis (16). Additionally, it has been described that TGF $\beta 1$-induced miR-1245 overexpression abolishes NKG2D, a receptor that participates in tumor immunosurveillance in natural killer (NK) cells, via binding to its 3'-UTR. Therefore, emerging evidence have suggested an oncogenic function of miR-1245a in several human malignancies, however, its role and clinical utility CRC had not been studied until recently $(17,18)$.

In the recent study, Pan et al. (19) explored, at molecular level, the biological role of miR-1245a in CRC, reporting its oncogenic function and the correlation of miR-1245a levels with poor prognosis of CRC patients. More specifically, the authors isolated total RNA from 20 colorectal tumors and paired adjacent non-cancerous colorectal tissue specimens in order to quantify miR-1245a expression. They have illustrated that miR-1245a was overexpressed in tumor specimens compared to adjacent non-malignant tissues, a notion which needs, however, further assessment due to the lack of relevant literature and the relatively small sample size analyzed. A more detailed description regarding the cycling and reaction protocol of the quantitative realtime polymerase chain reaction (qPCR) assay as well as the reference genes used for qPCR data normalization are considered necessary to achieve the greatest reproducibility of the presented results in future studies.
Subsequently, in order to assess the prognostic potential of miR-1245a in CRC, the authors downloaded publicly available data from The Cancer Genome Atlas (TCGA) and performed survival analysis by dichotomizing the cohort into high- and low- miR-1245a expressing patients, using the median as the optimal cutoff value. Concisely, KaplanMeier curves depicted that patients with elevated tumor miR-1245a levels succumb earlier than those expressing it at lower levels $(\mathrm{P}=0.0439)$. Moreover, multivariate Cox regression analysis has shown that expression of miR1245 a was an independent adverse prognosticator in CRC $(\mathrm{P}=0.039)$, namely patients with reduced miR-1245a tumor levels have an approximately 2 -fold increased cumulative survival probability. The potential role of miR-1245a as indicator of CRC progression is, beyond doubt, a key finding in the current study. However, we firmly believe that survival analysis using different clinical endpoints and stratified Kaplan-Meier curves according to histological grade of disease and/or TNM stage would be a more representative indicator of disease progression. As a result, miR-1245a could achieve a better risk stratification of CRC patients, strengthening, hence, its clinical utility and ameliorating disease clinical management. Apparently, a potential association of miR-1245a levels of patients suffering from stage II and stage III of the malignancy with prognosis would be very intriguing and aim the reduction of unwanted over- or undertreatment, since current biomarkers fail to discriminate low from high-risk patients in need for adjuvant chemotherapy and/or radiotherapy.

Thereafter, attempting to identify miR-1245a oncogenic mode of action in CRC, the authors performed colonyformation, cell proliferation (CCK8) and transwell invasion assays to demonstrate the increased proliferation and the significant invasive potential of miR-1245a-transfected cells, albeit the assessment was carried out using the miR1245a highly-expressing SW480 cell line. Subsequently, based on in silico target prediction, BRCA2 was uncovered as a candidate target gene of miR-1245a, and luciferase gene reporter assay further confirmed the direct binding in vitro. Ultimately, the miR-1245a-induced proliferative and invasive potential in vitro was hindered upon BRCA2 transfection, further validating their interaction. $B R C A 2$ gene encodes BRCA2 which is implicated in the repair mechanism of DNA double-strand breaks by homologous recombination. Specifically, BRCA2 is involved to the localization and binding ability of RAD51 recombinase at DNA damaged sites. Although, mutational status and epigenetic silencing of $B R C A 2$ are strongly associated with 
early-onset breast and ovarian cancers, their role in CRC needs yet to be elucidated. It is noteworthy that based on the current literature $B R C A 2$ deficiencies are not considered traditionally associated with CRC and do not confer in disease establishment except for some evidence regarding a potential oncogenic role of biallelic BRCA2 mutations in familial inheritance $(20,21)$.

The proposed mechanism of action of miR-1245a describes an increase of genomic instability mediated by a downregulation of $B R C A 2$, leading to enhanced proliferation and invasion of SW480 cells, which is in accordance with the results of previous studies in lung and breast cancer. As stated before, BRCA2 constitutes a key regulator in maintenance of genome integrity, however, to the best of our knowledge, deregulation of $B R C A 2$ has not been considered as a pivotal initiating event in CRC. On the contrary, a comprehensive report by Wang et al. (22) evaluating the prognostic significance of BRCA1/2 by immunohistochemistry in tumors of CRC patients revealed a significant higher overall survival (OS) for individuals with elevated BRCA2 protein levels. Taking into consideration all the aforementioned aspects, we speculate that downregulation of BRCA2 in CRC may represent a late event, which confers an increased risk for disease progression and/or lower survival probability, however, future studies will shed light on the actual role of BRCA2 in CRC biology.

In brief, the interesting study of Pan et al. (19) has reported the poor survival outcome of CRC patients characterized by miR-1245a overexpression as well as the post-transcriptional silencing of BRCA2 by miR-1245a, leading to the induction of cell proliferation and invasion in vitro, highlighting, thus, miR-1245a as biomarker and/or therapeutic target in CRC. However, for the comprehensive evaluation of miR-1245a and from bench to bedside processes, several limitations of the present research should be addressed and taken into consideration in future studies before conclusive results can be drawn. In this regard, the major limitation of the present study is the relatively small number of samples analyzed. Undoubtedly, future multiinstitutional large-scale studies, integrating miR-1245a with established molecular disease markers, would be of utmost interest to strengthen its prognostic significance and indicate the appropriate cutoff values to be exploited in molecular diagnostics. Besides, functional in vitro studies in a broader number of cell lines, investigating a variety of clinical endpoints, would be beneficial to unravel its significant implication in molecular pathways involved in cancer onset and progression and in vivo studies could further assess its role in personalized prognosis and tailored therapeutics.

\section{Acknowledgments}

Funding: The research presented was carried out within the framework of a Stavros Niarchos Foundation grant to the National and Kapodistrian University of Athens (grant ID 16785).

\section{Footnote}

Conflicts of Interest: All authors have completed the ICMJE uniform disclosure form (available at http://dx.doi. org/10.21037/atm-2020-113). The authors have no conflicts of interest to declare.

Ethical Statement: The authors are accountable for all aspects of the work in ensuring that questions related to the accuracy or integrity of any part of the work are appropriately investigated and resolved.

Open Access Statement: This is an Open Access article distributed in accordance with the Creative Commons Attribution-NonCommercial-NoDerivs 4.0 International License (CC BY-NC-ND 4.0), which permits the noncommercial replication and distribution of the article with the strict proviso that no changes or edits are made and the original work is properly cited (including links to both the formal publication through the relevant DOI and the license). See: https://creativecommons.org/licenses/by-nc-nd/4.0/.

\section{References}

1. Keum N, Giovannucci E. Global burden of colorectal cancer: emerging trends, risk factors and prevention strategies. Nat Rev Gastroenterol Hepatol 2019;16:713-32.

2. De Palma FDE, D'Argenio V, Pol J, et al. The Molecular Hallmarks of the Serrated Pathway in Colorectal Cancer. Cancers (Basel) 2019;11:1017.

3. Punt CJ, Koopman M, Vermeulen L. From tumour heterogeneity to advances in precision treatment of colorectal cancer. Nat Rev Clin Oncol 2017;14:235-46.

4. Consortium EP. An integrated encyclopedia of DNA elements in the human genome. Nature 2012;489:57-74.

5. Diamantopoulos MA, Tsiakanikas P, Scorilas A. Noncoding RNAs: the riddle of the transcriptome and their 
perspectives in cancer. Ann Transl Med 2018;6:241.

6. Avgeris M, Tsilimantou A, Levis PK, et al. Loss of GAS5 tumour suppressor lncRNA: an independent molecular cancer biomarker for short-term relapse and progression in bladder cancer patients. Br J Cancer 2018;119:1477-86.

7. Bartel DP. Metazoan MicroRNAs. Cell 2018;173:20-51.

8. Ameres SL, Zamore PD. Diversifying microRNA sequence and function. Nat Rev Mol Cell Biol 2013;14:475-88.

9. Ha M, Kim VN. Regulation of microRNA biogenesis. Nat Rev Mol Cell Biol 2014;15:509-24.

10. Panoutsopoulou K, Avgeris M, Scorilas A. miRNA and long non-coding RNA: molecular function and clinical value in breast and ovarian cancers. Expert Rev Mol Diagn 2018;18:963-79.

11. Hayes J, Peruzzi PP, Lawler S. MicroRNAs in cancer: biomarkers, functions and therapy. Trends Mol Med 2014;20:460-9.

12. Tsiakanikas P, Giaginis C, Kontos CK, et al. Clinical utility of microRNAs in renal cell carcinoma: current evidence and future perspectives. Expert Rev Mol Diagn 2018;18:981-91.

13. Avgeris M, Panoutsopoulou K, Papadimitriou MA, et al. Circulating exosomal miRNAs: clinical significance in human cancers. Expert Rev Mol Diagn 2019;19:979-95.

14. Kontos CK, Tsiakanikas P, Avgeris M, et al. miR15a-5p, A Novel Prognostic Biomarker, Predicting Recurrent Colorectal Adenocarcinoma. Mol Diagn Ther 2017;21:453-64.

Cite this article as: Scorilas A, Panoutsopoulou K, Tsiakanikas P. The interplay between miR-1245a and BRCA2 in colorectal cancer. Ann Transl Med 2020;8(17):1043. doi: 10.21037/atm-2020-113
15. Song L, Dai T, Xie Y, et al. Up-regulation of miR-1245 by c-myc targets BRCA2 and impairs DNA repair. J Mol Cell Biol 2012;4:108-17.

16. Yang L, Wang J, Fan Y, et al. Hsa_circ_0046264 upregulated BRCA2 to suppress lung cancer through targeting hsa-miR-1245. Respir Res 2018;19:115.

17. Espinoza JL, Nguyen VH, Ichimura H, et al. A functional polymorphism in the NKG2D gene modulates NK-cell cytotoxicity and is associated with susceptibility to Human Papilloma Virus-related cancers. Sci Rep 2016;6:39231.

18. Espinoza JL, Takami A, Yoshioka K, et al. Human microRNA-1245 down-regulates the NKG2D receptor in natural killer cells and impairs NKG2D-mediated functions. Haematologica 2012;97:1295-303.

19. Pan Z, Gan W, Liang C, et al. miR-1245a promotes the proliferation and invasion of colon adenocarcinoma by targeting BRCA2. Ann Transl Med 2019;7:777.

20. Prakash R, Zhang Y, Feng W, et al. Homologous recombination and human health: the roles of BRCA1, BRCA2, and associated proteins. Cold Spring Harb Perspect Biol 2015;7:a016600.

21. Degrolard-Courcet E, Sokolowska J, Padeano MM, et al. Development of primary early-onset colorectal cancers due to biallelic mutations of the FANCD1/BRCA2 gene. Eur J Hum Genet 2014;22:979-87.

22. Wang GH, Zhao CM, Huang Y, et al. BRCA1 and BRCA2 expression patterns and prognostic significance in digestive system cancers. Hum Pathol 2018;71:135-44. 